MATHEMATICS OF COMPUTATION

Volume 68, Number 226, April 1999, Pages 633-650

S $0025-5718(99) 01041-8$

\title{
ON THE ROBUSTNESS OF THE BPX-PRECONDITIONER WITH RESPECT TO JUMPS IN THE COEFFICIENTS
}

\author{
PETER OSWALD
}

\begin{abstract}
We determine the worst case behavior of the standard BPX-preconditioner for elliptic problems with arbitrary coefficient jumps along the boundaries of the coarsest partition. The counterexamples are also useful for other problems.
\end{abstract}

\section{INTRODUCTION}

Let $\Omega$ be a (bounded, open, connected) polyhedral domain in $\mathbb{R}^{d}$ equipped with an initial partition $\mathcal{T}_{0}=\left\{\Omega_{k}\right\}_{k=1}^{L}$ into $L \geq 2$ simplices. We restrict our attention to the cases $d=2,3$ of practical interest (although the statements below may be generalized to $d>3$ in a natural way). For convenience we also assume that $\mathcal{T}_{0}$ is quasi-uniform, with simplices of diameter $\asymp 1$. Let $\left\{\mathcal{T}_{j}\right\}_{j=0}^{J}$ be obtained by regular uniform dyadic refinement from $\mathcal{T}_{0}$. In particular, this means that analogous uniform refinement structures are inherited from $\left\{\mathcal{T}_{j}\right\}$ to all edges (and faces) of the $\Omega_{k} \in \mathcal{T}_{0}$, which is assumed without further mentioning.

Introduce the bilinear form

$$
a_{\omega}(u, v)=\sum_{k=1}^{L} \omega_{k}(\nabla u, \nabla v)_{L_{2}\left(\Omega_{k}\right)}, \quad u, v \in H_{0}^{1}(\Omega),
$$

with positive weights $\omega=\left\{\omega_{1}, \ldots, \omega_{L}\right\}$. As usual, we introduce the $H^{1}$ norm and seminorm with respect to any subdomain $\Omega_{k}$ by

$$
\|u\|_{1, \Omega_{k}}=\left(\|u\|_{L_{2}\left(\Omega_{k}\right)}^{2}+|u|_{1, \Omega_{k}}^{2}\right)^{1 / 2}, \quad|u|_{1, \Omega_{k}}=\|\nabla u\|_{L_{2}\left(\Omega_{k}\right)} .
$$

Thus,

$$
a_{\omega}(u, u)=\sum_{k=1}^{L} \omega_{k}|u|_{1, \Omega_{k}}^{2} \equiv|u|_{1, \omega}^{2} .
$$

We also need the weighted $L_{2}$ scalar product

$$
(u, v)_{0, \omega}=\sum_{k=1}^{L} \omega_{k}(u, v)_{L_{2}\left(\Omega_{k}\right)},
$$

Received by the editor July 12, 1996 and, in revised form, September 22, 1997.

1991 Mathematics Subject Classification. Primary 65N22, 65N55, 65F10.

Key words and phrases. Finite element multilevel preconditioners, robustness, elliptic problems with variable coefficients.

(C)1999 American Mathematical Society 
and the weighted $L_{2}$ and $H^{1}$ norms

$$
\|u\|_{0, \omega}=(u, u)_{0, \omega}^{1 / 2}, \quad\|u\|_{1, \omega}=\left(\|u\|_{0, \omega}^{2}+|u|_{1, \omega}^{2}\right)^{1 / 2}
$$

corresponding to $\omega$.

We consider the finite element approximation of the variational problem associated with $a_{\omega}(\cdot, \cdot)$ on $H_{0}^{1}(\Omega)$. Throughout the paper, we denote by $S_{1}^{0}(\mathcal{T})$ the set of linear finite element functions with respect to a partition $\mathcal{T}$. Thus,

$$
V_{j}=S_{1}^{0}\left(\mathcal{T}_{j}\right) \cap H_{0}^{1}(\Omega), \quad j=0, \ldots, J,
$$

will be the discretization spaces of interest. The standard nodal basis functions in $S_{1}^{0}\left(\mathcal{T}_{j}\right)$ are denoted by $\phi_{j, i}$; the subset of those $\phi_{j, i}$ vanishing at $\partial \Omega$ forms the basis in $V_{j}$. The problem of determining $u \in V_{J}$ such that

$$
a_{\omega}(u, v)=\langle f, v\rangle_{H^{-1} \times H_{0}^{1}} \quad \forall v \in V_{J}
$$

leads to a large sparse system of equations. In the case $\omega_{1}=\ldots=\omega_{L}$, one of the most popular (and efficient) methods for solving this system iteratively is the preconditioned conjugate gradient method with the multilevel BPX-preconditioner proposed by Bramble, Pasciak, and $\mathrm{Xu}$ [3]. In the weighted case (arbitrary $\omega$ ), Dryja, Sarkis, and Widlund $[5,6]$ have established the optimality of this preconditioner under special assumptions on $\omega$. It is folklore that this method is not robust with respect to general $\omega$. See $[4,11]$ for the closely related problem of robustness of weighted $L_{2}$ approximation. However, no definitive results seem to be available so far. It is the aim of this note to answer this question.

As is well-known $[12,14,7,9]$, the above-mentioned BPX-preconditioner is mathematically determined by the additive Schwarz operator

$$
P_{\omega, J} u=P_{\omega, 0} u+\sum_{j=1}^{J} \sum_{i} \frac{a_{\omega}\left(u, \phi_{j, i}\right)}{a_{\omega}\left(\phi_{j, i}, \phi_{j, i}\right)} \phi_{j, i}
$$

where $P_{\omega, 0} u \in V_{0}$ solves the problem

$$
a_{\omega}\left(P_{\omega, 0} u, v_{0}\right)=a_{\omega}\left(u, v_{0}\right) \quad \forall v_{0} \in V_{0} .
$$

If not indicated otherwise, $\sum_{i}$ means summation with respect to all basis functions in $V_{i}$. The associated subspace splitting (see [7] for the terminology and for a survey of basic results on additive Schwarz operators) is

$$
\left\{V_{J} ; a_{\omega}\right\}=\left\{V_{0} ; a_{\omega}\right\}+\sum_{j=1}^{J} \sum_{i}\left\{V_{j, i} ; a_{\omega}\right\},
$$

with the one-dimensional subspaces $V_{j, i}$ spanned by the individual basis functions $\phi_{j, i}$. The operator $P_{\omega, J}: V_{J} \rightarrow V_{J}$ is symmetric positive definite with respect to $a_{\omega}(\cdot, \cdot)$, and the preconditioning power of the BPX-preconditioner is described by the numbers

$$
\kappa\left(P_{\omega, J}\right)=\frac{\lambda_{\max }}{\lambda_{\min }}, \quad \lambda_{\max }=\max _{0 \neq u \in V_{J}} \frac{a_{\omega}(u, u)}{\|\| u \|_{\omega}^{2}}, \quad \lambda_{\min }=\min _{0 \neq u \in V_{J}} \frac{a_{\omega}(u, u)}{\|\| u \mid \|_{\omega}^{2}},
$$

see [7, Theorem 16]. The triple bar norm is given by

$$
\|\| u \|\left.\right|_{\omega} ^{2}=\inf _{u_{0} \in V_{0}, u_{j, i} \in V_{j, i}: u=u_{0}+\sum_{j=1}^{J} \sum_{i} u_{j, i}} a_{\omega}\left(u_{0}, u_{0}\right)+\sum_{j=1}^{J} \sum_{i} a_{\omega}\left(u_{j, i}, u_{j, i}\right) .
$$




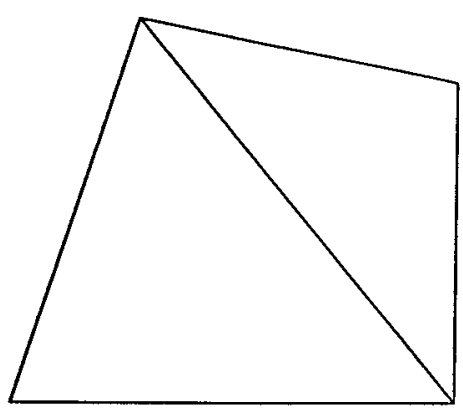

a)

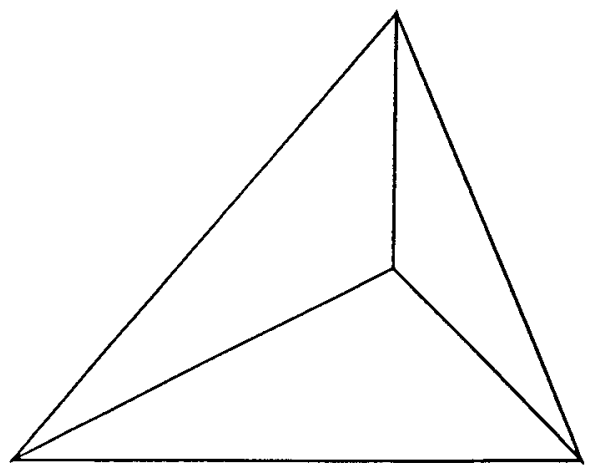

b)

Figure 1. Exceptional $\mathcal{T}_{0}: d=2$

We state the main results of this note separately for $d=2$ and $d=3$. For $d=2$, we call a triangulation $\mathcal{T}_{0}$ exceptional if it is equivalent to one of the two triangulations shown in Figure 1.

Theorem 1. Let $d=2$. If $\mathcal{T}_{0}$ is not exceptional then

$$
\kappa_{J} \equiv \sup _{\omega} \kappa\left(P_{\omega, J}\right) \asymp J^{2}, \quad J \rightarrow \infty .
$$

For the two exceptional $\mathcal{T}_{0}$, the sharper estimate $\kappa_{J}=\mathrm{O}(1), J \rightarrow \infty$, holds true. The constants are independent of $J$ but may depend on $\mathcal{T}_{0}$.

In (7) and throughout the paper, $\asymp$ stands for a two-sided inequality between the expressions, with positive constants that do not depend on the parameters involved. Roughly speaking, Theorem 1 states that for the jumping coefficient case the BPXpreconditioner has the same worst case behavior as the hierarchical basis method of Yserentant [13]. The upper estimate in (7) follows from [4, Theorem 4.5] and for the exceptional cases from [5, Theorem 3] (more precisely, by its two-dimensional analog, see $[6$, section 5]). The counterexamples which show the lower bound in (7) for the non-exceptional case seem to be new; however, analogous examples have been used in $[13,8]$. In particular, they show that the result of [10, Theorem 4.2] is incorrect for $\mathcal{T}_{0}$ with interior points.

The case $d=3$ is a bit more complicated. We consider three types of initial partitions. We say that $\mathcal{T}_{0}$ is of point type if there exists at least one tetrahedron $\Omega_{k_{0}}$ such that $\partial \Omega_{k_{0}} \cap \partial \Omega$ contains no edges and at least one (boundary) vertex. $\mathcal{T}_{0}$ is exceptional if it is equivalent to one of the three initial partitions shown in Figure 2. All other $\mathcal{T}_{0}$ are said to be of edge type. It turns out that in an initial partition of edge type all $\Omega_{k}$ should touch the boundary $\partial \Omega$ at least along an edge which justifies the name. We derive this and further geometric properties of $\mathcal{T}_{0}$ in section 2 .

Theorem 2. Let $d=3$. For $\kappa_{J}$ as defined in (7) we have

$$
\kappa_{J} \asymp\left\{\begin{array}{ll}
2^{J} & \text { if } \mathcal{T}_{0} \text { is of point type, } \\
J^{2} & \text { if } \mathcal{T}_{0} \text { is of edge type, } \\
1 & \text { if } \mathcal{T}_{0} \text { is exceptional, }
\end{array} \quad J \rightarrow \infty .\right.
$$

The constants are independent of $J$ but may depend on $\mathcal{T}_{0}$. 


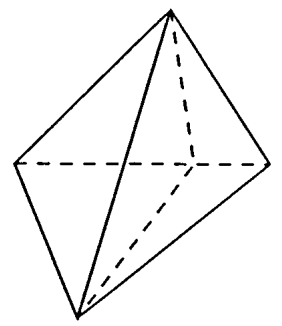

a)

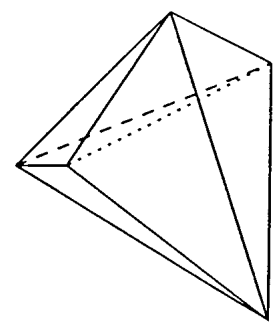

b)

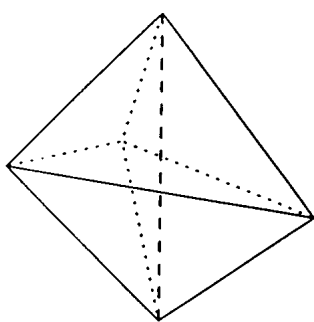

c)

Figure 2. Exceptional $\mathcal{T}_{0}: d=3$

The upper estimates are essentially known, at least for exceptional $\mathcal{T}_{0}$ and for $\mathcal{T}_{0}$ of point type, where they follow from [5, 6] and [4], respectively. The counterexamples for the latter case are similar to those used in [11]. The result on the asymptotic behaviour of $\kappa_{J}$ for $\mathcal{T}_{0}$ of edge type is partly new. Here, the counterexamples are inherited from the two-dimensional case.

The statements of Theorems 1 and 2 have extensions to the case when the subdomains $\Omega_{k}$ are unions of several simplices of $\mathcal{T}_{0}$ as considered in [4], to other element types, other boundary conditions, and fourth order elliptic problems. An extension to dimensions $d \geq 4$ is also possible. Since these extensions only add technical difficulties but do not exhibit principally new phenomena, we omit them here.

Analogous results hold for the worst case behavior of the multiplicative multilevel Schwarz iteration associated with the splitting $(4)$ (see $[2,11]$ for a description of this algorithm which corresponds to a simple $V$-cycle multigrid method). This follows from the two-sided estimates for the norms of the iteration operators governing the multiplicative method (compare, e.g., [15, Theorem 5.1]) and the fact that the deterioration of $\kappa\left(P_{\omega, J}\right)$ is due to the deterioration of $\lambda_{\min }$ while $\lambda_{\max } \asymp 1$ (and the corresponding Cauchy-Schwarz inequalities) hold uniformly for all $\omega>0$. Details will not be discussed in this note. The estimations of $\lambda_{\min }$ and $\lambda_{\max }$ can be found in the next section.

The asymptotically exact worst case estimate of Theorems 1 and 2 is complemented by the trivial estimate

$$
\kappa\left(P_{\omega, J}\right) \leq C \frac{\max _{k} \omega_{k}^{2}}{\min _{k} \omega_{k}^{2}},
$$

which comes from switching to the case of constant weights (see [7, Theorem 19] for the uniform condition number estimate if $\omega_{1}=\ldots=\omega_{L}$ ), and by the results of $[5,6]$ on so-called quasimonotone weights $\omega$. The counterexamples are of interest also in connection with $[4,11]$ (see the end of section 3) and with other inequalities for (weighted) discrete norms of finite element functions and their traces. For example, they may be used to show the asymptotic sharpness of the convergence results for various domain decomposition methods based on substructuring. 


\section{UPPER ESTIMATES}

We start by introducing some notation. Let $\Omega_{j, i}$ denote the (open) support of $\phi_{j, i}$, and set

$$
\omega_{j, i}=\max \left\{\omega_{k}: \Omega_{k} \cap \Omega_{j, i} \neq \emptyset\right\} .
$$

Obviously, by the properties of the nodal bases $\left\{\phi_{j, i}\right\}$

$$
\begin{aligned}
\|u\| \|_{\omega}^{2} & \asymp \operatorname{unf}_{u_{0} \in V_{0}, u_{j, i} \in V_{j, i}: u=u_{0}+\sum_{j=1}^{J} \sum_{i} u_{j, i}}\left(a_{\omega}\left(u_{0}, u_{0}\right)+\sum_{j=1}^{J} \sum_{i} \omega_{j, i} 2^{2 j}\left\|u_{j, i}\right\|_{L_{2}}^{2}\right) \\
(9) & \asymp \inf _{u=u_{0}+\sum_{j} \sum_{i} u_{j, i}}\left(a_{\omega}\left(u_{0}, u_{0}\right)+\sum_{j=1}^{J} \sum_{k=1}^{L} \omega_{k} \sum_{i: \Omega_{j, i} \cap \Omega_{k} \neq \emptyset} 2^{2 j}\left\|u_{j, i}\right\|_{L_{2}\left(\Omega_{k}\right)}^{2}\right) \\
(10) & \asymp \inf _{u_{j} \in V_{j}: u=\sum_{j=0}^{J} u_{j}}\left(a_{\omega}\left(u_{0}, u_{0}\right)+\sum_{j=1}^{J} 2^{2 j}\left\|u_{j}\right\|_{0, \omega}^{2}\right) \equiv\|u\| \|_{0, \omega}^{2} .
\end{aligned}
$$

These equivalent representations (up to constants) of the triple bar norm $\left|\|\cdot \mid\|_{\omega}\right.$ are very convenient. The discrete version (9) will be explored in connection with the counterexamples of section 3 . As a choice for the $u_{j}$ which realizes the infimum (up to constants) in (10), one should think of $u_{j}=Q_{j, \omega} u-Q_{j-1, \omega} u$, with $Q_{j, \omega}$ denoting the $L_{2, \omega}$ orthogonal projection operators into $V_{j}$. This makes the connection with $[4,11]$ more transparent. Below, the terms in the sum (10) will be further split with respect to interior vertices, edges, and faces, so that the weights are constant in each group. This leads to the study of a variety of "local", unweighted triple bar norms with restrictions on the set of admissible $u_{j, i}$ (a closer look at the restrictions to be expected shows that all this is closely related to the study of norms for finite element functions with trace restrictions). The following lemma contains the basic theoretical result on the BPX-preconditioner in the non-weighted case (see, e.g., [7, Theorem 15]).

Lemma 1. For arbitrary $d \geq 1$, let $\Omega,\left\{\mathcal{T}_{j}\right\},\left\{V_{j}\right\}$ be as defined above. Then, for any $u \in V_{J}$,

$$
\|u\|_{H^{1}(\Omega)}^{2} \asymp\left|\left\|u | | ^ { 2 } \asymp \left|\left\|u | | _ { 0 } ^ { 2 } \asymp | \| u \| | _ { * } ^ { 2 } \asymp \left|\left\|\left.u||_{b a}^{2} \asymp|\| u|\right|_{Q} ^{2},\right.\right.\right.\right.\right.\right.
$$

where \|\|$u\|\|^{2}$ and \|\|$u\|\|_{0}^{2}$ are defined as in (6) and (10), respectively, but with uniform weight $\omega=\{1,1, \ldots, 1\}$, and

$$
\begin{aligned}
\|u\|_{*}^{2} & =\inf _{u_{j} \in V_{j}: u=\sum_{j=1}^{J} u_{j}} \sum_{j=1}^{J} 2^{2 j}\left\|u_{j}\right\|_{L_{2}(\Omega)}^{2}, \\
\|\| u \|_{b a}^{2} & \equiv\|u\|_{L_{2}(\Omega)}^{2}+\sum_{j=1}^{J-1} 2^{2 j} E_{j}(u)_{L_{2}(\Omega)}^{2}, \\
\|\| u \|_{Q}^{2} & \equiv\left\|Q_{0} u\right\|_{L_{2}(\Omega)}^{2}+\sum_{j=1}^{J} 2^{2 j}\left\|Q_{j} u-Q_{j-1} u\right\|_{L_{2}(\Omega)}^{2} .
\end{aligned}
$$

Here,

$$
E_{j}(u)_{L_{2}(\Omega)}=\inf _{v_{j} \in V_{j}}\left\|u-v_{j}\right\|_{L_{2}(\Omega)}, \quad j=0, \ldots, J-1
$$


denotes the best $L_{2}$ approximation of $u$, and $Q_{j}$ the $L_{2}(\Omega)$ orthoprojection onto $V_{j}$. The constants in the norm equivalences (11) depend only on the shape of the simplices in $\mathcal{T}_{0}$ but not on $u$ and $J$. The result also holds if $V_{j}=S_{1}^{0}\left(\mathcal{T}_{j}\right) \cap H_{0}^{1}(\Omega)$ is replaced by $V_{j}=S_{1}^{0}\left(\mathcal{T}_{j}\right)$.

The equivalence of the triple bar norm (12) with (13) and (14) holds under fairly general conditions (a general Hilbert space norm instead of the $L_{2}$ norm and any increasing sequence of subspaces $V_{j}$ ) as long as the weighting factors continue to grow at a geometric rate. This will occasionally be used below. Note that the summation in (12) and (13) could also include $j=0$, without changing the norm equivalence. Since $V_{0}$ contains constant functions, estimates for the $H^{1}$ seminorm can also be deduced for the choice $V_{j}=S_{1}^{0}\left(\mathcal{T}_{j}\right)$.

For general $\omega$ and arbitrary $u \in V_{J}$, by locally applying Lemma 1 with the triple bar norm (12) to $\left.\left.\left(u-u_{0}\right)\right|_{\Omega_{k}} \in V_{J, \Omega_{k}} \equiv V_{J}\right|_{\Omega_{k}}$ on the simplices $\Omega_{k}$ we obtain

$$
\begin{aligned}
a_{\omega}(u, u) \leq & C \inf _{u_{0} \in V_{0}}\left(a_{\omega}\left(u_{0}, u_{0}\right)+\sum_{k} \omega_{k}\left|u-u_{0}\right|_{1, \Omega_{k}}^{2}\right) \\
\leq & C \inf _{u_{0} \in V_{0}}\left\{a_{\omega}\left(u_{0}, u_{0}\right)\right. \\
& \left.\quad+\sum_{k=1}^{L} \omega_{k} \inf _{u_{j, \Omega_{k}} \in V_{j, \Omega_{k}}: u-u_{0} \mid \Omega_{k}=\sum_{j=1}^{J} u_{j, \Omega_{k}}} \sum_{j=1}^{J} 2^{2 j}\left\|u_{j, \Omega_{k}}\right\|_{L_{2}\left(\Omega_{k}\right)}^{2}\right\} \\
\leq & C \inf _{u_{j} \in V_{j}: u=u_{0}+\sum_{j=1}^{J} u_{j}}\left(a_{\omega}\left(u_{0}, u_{0}\right)+\sum_{j=1}^{J} 2^{2 j}\left\|u_{j}\right\|_{0, \omega}^{2}\right) \\
& =C\left|\left\|u \left|\left\|_{0, \omega}^{2} \leq C|\| u|_{\omega}^{2},\right.\right.\right.\right.
\end{aligned}
$$

compare (10). From these inequalities we have

$$
\lambda_{\max } \leq C,
$$

independently of the weights $\omega$ and $J$ (see (5) for the definition of $\lambda_{\max }$ ). Since

$$
\left\|\phi_{j, i} \mid\right\|_{\omega}^{2} \leq a_{\omega}\left(\phi_{j, i}, \phi_{j, i}\right)
$$

for any basis function of level $j \geq 1$ by definition of the triple bar norm, we arrive at

Lemma 2. For $\lambda_{\max }$ as defined in (5) we have

$$
\lambda_{\max } \asymp 1,
$$

with constants depending on $\mathcal{T}_{0}$ and $d$ but independent of $\omega$ and $J$.

The remainder of this section consists of proving robust lower estimates for $\lambda_{\min }$ which together with Lemma 2 lead to the upper estimates for $\kappa_{J}$ in Theorem 1 . In most cases, these bounds follow from the following estimates for $L_{2, \omega}$ orthoprojections which have essentially been obtained in [4].

Theorem 3. Let $d=2,3$, and denote by $Q_{j, \omega}: V_{J} \rightarrow V_{j}$ the orthogonal projection with respect to the $L_{2, \omega}$ scalar product (2). Then

$$
a_{\omega}\left(Q_{j, \omega} u, Q_{j, \omega} u\right)+2^{2 j}\left\|u-Q_{j, \omega} u\right\|_{L_{2, \omega}(\Omega)}^{2} \leq C a_{\omega}(u, u) \cdot \begin{cases}J-j+1, & d=2, \\ 2^{(J-j)}, & d=3 .\end{cases}
$$


with constants independent of $j=0, \ldots, J-1, u \in V_{J}, J$, and $\omega$. If $d=3$, and $\mathcal{T}_{0}$ is of edge type, then (16) can be replaced by the improved estimate

$$
a_{\omega}\left(Q_{j, \omega} u, Q_{j, \omega} u\right)+2^{2 j}\left\|u-Q_{j, \omega} u\right\|_{L_{2, \omega}(\Omega)}^{2} \leq C J a_{\omega}(u, u) .
$$

Except for (17), Theorem 3 is contained in [4, Theorem 4.5]. As observed in [4, Remark 3.1], the estimate for $a_{\omega}\left(Q_{j, \omega} u, Q_{j, \omega} u\right)$ follows from the $L_{2, \omega}$ error bound for $Q_{j, \omega}$. The proof of (17) will be given at the end of this section.

Together with (10), Theorem 3 immediately gives

$$
\begin{aligned}
\|\| u \|_{\omega}^{2} & \leq C\left(a_{\omega}\left(Q_{0, \omega} u, Q_{0, \omega} u\right)+\sum_{j=1}^{J} 2^{2 j}\left\|Q_{j, \omega} u-Q_{j-1, \omega} u\right\|_{L_{2, \omega}(\Omega)}^{2}\right) \\
& \leq C\left(a_{\omega}\left(Q_{0, \omega} u, Q_{0, \omega} u\right)+\sum_{j=0}^{J} 2^{2 j}\left\|u-Q_{j, \omega} u\right\|_{L_{2, \omega}(\Omega)}^{2}\right) \\
& \leq C a_{\omega}(u, u) \cdot\left\{\begin{array}{l}
\sum_{j=0}^{J} J \quad \leq C a_{\omega}(u, u) \cdot\left\{\begin{array}{l}
J^{2}, \\
\sum_{j=0}^{J} 2^{(J-j)}
\end{array}\right.
\end{array}\right.
\end{aligned}
$$

where the first case applies to $d=2$ and to $\mathcal{T}_{0}$ of edge type if $d=3$, and the second to general $\mathcal{T}_{0}$ if $d=3$. For the exceptional domains of Figures 1 and 2, one observes that any weight $\omega$ is quasimonotone in the terminology of $[5,6]$, which gives a better $\mathrm{O}(1)$ bound by applying [5, Theorem 3]. We arrive at

Lemma 3. For $\lambda_{\min }$ as defined in (5) we have

$$
\left(\lambda_{\text {min }}\right)^{-1} \leq C \cdot\left\{\begin{array}{l}
1, \\
J^{2}, \\
2^{J},
\end{array}\right.
$$

where the first case applies to the five exceptional $\mathcal{T}_{0}$, the second to all remaining initial partitions for $d=2$ and to all $\mathcal{T}_{0}$ of edge type for $d=3$, and the third to $\mathcal{T}_{0}$ of point type for $d=3$. The constants in (18) depend on $\mathcal{T}_{0}$ and $d$ but are independent of $\omega$ and $J$.

The results of Lemma 2 and 3 imply the upper estimates for $\kappa_{J}$ in Theorems 1 and 2 in all cases. It remains to provide a proof of (17). To this end, we need some geometric properties of initial partitions $\mathcal{T}_{0}$ which will be useful also in connection with the construction of counterexamples in section 3. Let $\Gamma_{k}=\partial \Omega_{k} \cap \partial \Omega$ and notice that $\Gamma_{k}$ either is empty or decomposes into closed connected components which represent single vertices (point components) or unions of $\geq 1$ edges (edge components) or unions of $\geq 1$ faces (face components) or, finally, unions of edges and faces (mixed components). The latter two types appear only if $d=3$.

Lemma 4. a) Let $d=2$. Then any $\mathcal{T}_{0}$ satisfies at least one of the following properties:

$(\alpha) \mathcal{T}_{0}$ is exceptional.

( $\beta)$ There is at least one triangle $\Omega_{k}$ for which $\Gamma_{k}$ contains a point component $\{P\}$.

$(\gamma) \mathcal{T}_{0}$ consists of at least 4 triangles $\Omega_{k}$ that all share a single interior vertex $P$ of $\mathcal{T}_{0}$ while the opposite edge is on $\partial \Omega$. 


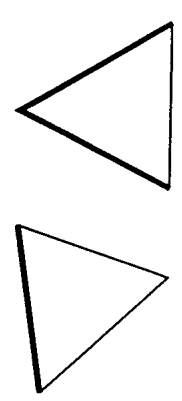

a)

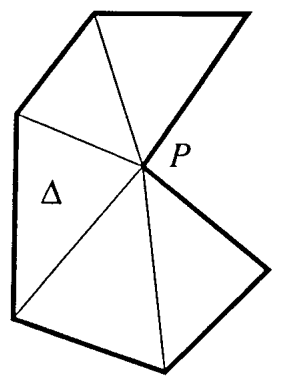

b)

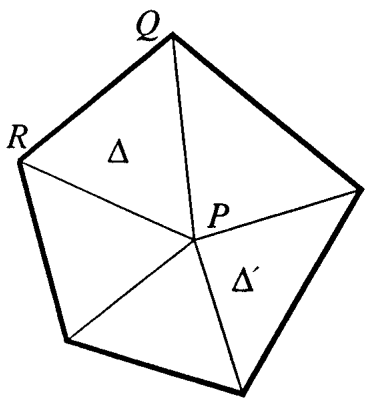

c)

FiguRE 3. Illustrations for the case that $(\beta)$ is violated

b) Let $d=3$, and let $\mathcal{T}_{0}$ be of edge type. Then each $\Gamma_{k}$ contains at least one edge. Moreover, at least one of the following properties holds:

$(\delta)$ There is a tetrahedron $\Omega_{k}$ with two interior faces that meet at a boundary edge.

$(\epsilon)$ There is at least one interior edge with 4 or more tetrahedra $\Omega_{k}$ attached to it.

Proof. Let $d=2$, and assume that $(\beta)$ is not satisfied. This means that for all boundary triangles $\Gamma_{k}$ consists of one edge or the union of two edges; see Figure 3 a). Now observe that, when starting at any boundary triangle and moving to its neighbors, we arrive after finitely many steps at one of the situations shown in Figure $3 \mathrm{~b}$ ) (a "chain" of triangles with no interior vertex which is of exceptional type, Figure 1 a), or satisfies $(\beta)$, i.e. contradicts our assumption) or in Figure 3 c) (a triangulation with one interior vertex which is exceptional, Figure $1 \mathrm{~b}$ ), or satisfies $(\gamma))$. This proves the statement.

Now let $d=3$. Assume that $\mathcal{T}_{0}$ is not of point type. Thus, any boundary tetrahedron $\left(\Gamma_{k} \neq \emptyset\right)$ contains at least one boundary edge. But this immediately implies that there are no interior tetrahedra $\Gamma_{k}=\emptyset$ in $\mathcal{T}_{0}$. Thus, all $\Omega_{k}$ are boundary tetrahedra, and $\Gamma_{k}$ contains at least one edge.

Suppose that $(\delta)$ does not hold. A moment's reflection shows that then for any $\Omega_{k}$ the set $\Gamma_{k}$ is the union of one or more faces. Assume next that $\mathcal{T}_{0}$ possesses at least one interior vertex, which we denote by $P$. In this case $\mathcal{T}_{0}$ represents the three-dimensional analog of the triangulation of Figure $3 \mathrm{c}$ ), since for any $\Omega_{k}$ with $P$ as a vertex, the boundary set $\Gamma_{k}$ must be the face opposite to $P$. Thus, $\mathcal{T}_{0}$ is either exceptional, Figure $2 \mathrm{c}$ ), or satisfies $(\epsilon)$ (to see the latter, consider the triangulated boundary of $\Omega$ and observe that $(\epsilon)$ is equivalent to finding one vertex of this boundary triangulation with at least 4 triangles attached).

On the other hand, if there is no interior vertex then $\Gamma_{k}$ is the union of at least 2 faces for all $k$. The case of 4 faces in some $\Gamma_{k}$, i.e., $\Gamma_{k}=\partial \Omega_{k}$, contradicts the connectedness of $\Omega$ or $L \geq 2$. If some $\Gamma_{k}$ contains 3 faces then the only reasonable partition (satisfying all other requested properties) is the exceptional one of Figure 2 a). Thus, we can assume that $\Gamma_{k}$ is the union of exactly 2 faces for all $k$. Once again, starting with any of the $\Omega_{k}$ and going from neighbour to neighbour through interior faces, we see that in this case $\mathcal{T}_{0}$ is characterized by having exactly one 
interior edge, with the $\Omega_{k}$ "winding" around it. Again, this results in an exceptional partition $(L=3$, Figure $2 \mathrm{~b})$ ) or a partition satisfying $(\epsilon)$.

We come to the proof of (17). The following notation is used: $Q_{j, \Omega_{k}}, Q_{j, f}, Q_{j, e}$ denote the (unweighted) $L_{2}\left(\Omega_{k}\right), L_{2}(f), L_{2}(e)$ orthoprojections from $V_{J, \Omega_{k}}, V_{J, f} \equiv$ $\left.S_{1}^{0}\left(\mathcal{T}_{J}\right)\right|_{f},\left.V_{J, e} \equiv S_{1}^{0}\left(\mathcal{T}_{J}\right)\right|_{e}$ into $V_{j, \Omega_{k}}, V_{j, f}, V_{j, e}$ corresponding to the tetrahedra $\Omega_{k}$ of $\mathcal{T}_{0}$, their faces $f$, and edges $e$, respectively. For arbitrary $u \in V_{J}$, let $u_{j} \in V_{j}$ be defined at the nodal points $P$ of $\mathcal{T}_{j}$ by

$$
u_{j}(P)= \begin{cases}Q_{j, \Omega_{k}} u(P), & P \in \operatorname{int}\left(\Omega_{k}\right), \\ Q_{j, f} u(P), & P \in \operatorname{int}(f), \\ Q_{j, e} u(P), & P \in \operatorname{int}(e), \\ 0, & \text { otherwise }\end{cases}
$$

It is straightforward (see [4] for quite analogous considerations) that

$$
\begin{aligned}
& \left\|u-u_{j}\right\|_{L_{2}\left(\Omega_{k}\right)}^{2} \leq 2\left(\left\|u-Q_{j, \Omega_{k}} u\right\|_{L_{2}\left(\Omega_{k}\right)}^{2}+\left\|Q_{j, \Omega_{k}} u-u_{j}\right\|_{L_{2}\left(\Omega_{k}\right)}^{2}\right) \\
& \leq \quad C\left(\left\|u-Q_{j, \Omega_{k}} u\right\|_{L_{2}\left(\Omega_{k}\right)}^{2}+2^{-j} \sum_{f \subset \partial \Omega_{k}}\left\|Q_{j, \Omega_{k}} u-u_{j}\right\|_{L_{2}(f)}^{2}\right) \\
& \leq \quad C\left(\left\|u-Q_{j, \Omega_{k}} u\right\|_{L_{2}\left(\Omega_{k}\right)}^{2}\right. \\
& \left.+2^{-j} \sum_{f \subset \partial \Omega_{k}}\left(\left\|Q_{j, \Omega_{k}} u-Q_{j, f} u\right\|_{L_{2}(f)}^{2}+\left\|Q_{j, f} u-u_{j}\right\|_{L_{2}(f)}^{2}\right)\right) \\
& \quad \ldots \quad \sum_{j, \Omega_{k}} u\left\|_{L_{2}\left(\Omega_{k}\right)}^{2}+\sum_{f \subset \partial \Omega_{k}} 2^{-j}\right\| Q_{j, f}\left(u-Q_{j, \Omega_{k}} u\right) \|_{L_{2}(f)}^{2} \\
& \left.\quad+2^{-2 j} \sum_{f \subset \partial \Omega_{k}} \sum_{e \in \partial f}\left\|Q_{j, e}\left(u-Q_{j, f} u\right)\right\|_{L_{2}(e)}^{2}+2^{-3 j} \sum_{e \subset \partial \Omega_{k}} \sum_{P \subset \partial e} Q_{j, e} u(P)^{2}\right) \\
& \leq \quad C\left(\left\|u-Q_{j, \Omega_{k}} u\right\|_{L_{2}\left(\Omega_{k}\right)}^{2}+\sum_{f \subset \partial \Omega_{k}} 2^{-j}\left\|u-Q_{j, \Omega_{k}}\right\|_{L_{2}(f)}^{2}\right. \\
& \left.\quad+2^{-2 j} \sum_{f \subset \partial \Omega_{k}} \sum_{e \in \partial f}\left\|u-Q_{j, f} u\right\|_{L_{2}(e)}^{2}+2^{-2 j} \sum_{e \subset \partial \Omega_{k}}\left\|Q_{j, e} u\right\|_{L_{2}(e)}^{2}\right) .
\end{aligned}
$$

The first three terms (associated with the projections $Q_{j, \Omega_{k}}$ and $Q_{j, f}$ ) can be estimated by $C 2^{-2 j}|u|_{1, \Omega_{k}}^{2}$ and $C J 2^{-2 j}|u|_{1, \Omega_{k}}^{2}$ in a standard way, using the results in [4] or Lemma 1. We leave the details to the reader. Only for estimating the terms $\left\|Q_{j, e} u\right\|_{L_{2}(e)}^{2}, e \in \partial \Omega_{k}$, we need that $\mathcal{T}_{0}$ is of edge type. By Lemma 4 , there is another edge $e^{\prime}$ of $\Omega_{k}$ such that $u \equiv 0$ on $e^{\prime}$ (if $e=e^{\prime}$, the above integral vanishes). Let

$$
v_{l}=Q_{l, \Omega_{k}} u, \quad l \geq 1, \quad v_{0}=\bar{u}_{\Omega_{k}} \equiv\left(\operatorname{meas} \Omega_{k}\right)^{-1} \int_{\Omega_{k}} u d x
$$


and $u_{l}=\left.\left(v_{l}-v_{l-1}\right)\right|_{e}, \tilde{u}_{l}=\left(v_{l}-v_{l-1}\right)_{e^{\prime}}$. With this notation,

$$
\begin{aligned}
\left\|Q_{j, e} u\right\|_{L_{2}(e)}^{2} & \leq\|u\|_{L_{2}(e)}^{2} \leq C\left(\bar{u}_{\Omega_{k}}^{2}+J \sum_{l=1}^{J}\left\|u_{l}\right\|_{L_{2}(e)}^{2}\right) \\
& \leq C\left(\left\|-\sum_{l=1}^{J} \tilde{u}_{l}\right\|_{L_{2}\left(e^{\prime}\right)}^{2}+J \sum_{l=1}^{J}\left\|u_{l}\right\|_{L_{2}(e)}^{2}\right) \\
& \leq C J\left(\sum_{l=1}^{J}\left\|\tilde{u}_{l}\right\|_{L_{2}\left(e^{\prime}\right)}^{2}+\sum_{l=1}^{J}\left\|u_{l}\right\|_{L_{2}(e)}^{2}\right) \\
& \leq C J \sum_{l=1}^{J} 2^{2 l}\left\|v_{l}-v_{l-1}\right\|_{L_{2}\left(\Omega_{k}\right)}^{2} \leq C J|u|_{1, \Omega_{k}}^{2}
\end{aligned}
$$

compare the equivalence with (14) in Lemma 1 for the last estimation step. Taking all estimates together, we arrive at (17), which also completes the proof of Theorem 3 and Lemma 3.

\section{Counterexamples}

The proof of the sharpness of the bound (18) for $\lambda_{\min }$ requires the following technical result (see [7, p.89/90] or [9, p.11]) for partial results). Since there might be other applications, we formulate it for general $d \geq 1$ although it essentially reduces to a one-dimensional result. Let us fix any simplex $\Omega^{*}$ from $\mathcal{T}_{0}$ and its vertex $P$ (for the following to be formally correct, it should be assumed that all (open) faces of $\Omega^{*}$ attached to $P$ are in the interior of $\Omega$ ). The basis function $\phi_{j, i} \in S_{1}^{0}\left(\mathcal{T}_{j}\right)$ satisfying $\phi_{j, i}(P)=1$ will be denoted by $\phi_{j, P}, j=0, \ldots, J$. For later convenience we set

$$
I_{P, \Omega^{*}}=\left\{(j, i): \phi_{j, i} \in V_{j}, \Omega_{j, i} \cap \Omega^{*} \neq \emptyset, \phi_{j, i}(P)=0\right\},
$$

and $V_{j}^{*}=\operatorname{span}\left\{\phi_{j, i}:(j, i) \in I_{P, \Omega^{*}}\right\}, j=0, \ldots, J$. For ease in understanding the technicalities below, the reader may restrict his attention to the case where $\mathcal{T}_{0}$ consists of one simplex $\Omega_{1}=\Omega=\Omega^{*}$ and $V_{j}=S_{1}^{0}\left(\mathcal{T}_{j}\right)$. In that case $I_{P, \Omega^{*}}$ contains all index pairs but those corresponding to the $\phi_{j, P}$, and $V_{j}^{*}=\left\{u_{j} \in V_{j}: u_{j}(P)=\right.$ $0\}$.

Lemma 5. Let $d \geq 1$. For any

$$
u_{P}=\sum_{j=0}^{J} c_{j} \phi_{j, P}
$$

we have

$$
\left|u_{P}\right|_{1, \Omega^{*}}^{2} \asymp\left\|u_{P}\right\|_{1, \Omega^{*}}^{2} \asymp \sum_{j=0}^{J} c_{j}^{2} 2^{-j(d-2)} .
$$

If in addition $u_{P}(P)=0$, then

$$
\left\|u_{P}||_{P, \Omega^{*}}^{2} \equiv \inf _{\left.u_{P}\right|_{\Omega^{*}}=\left.\sum_{(j, i) \in I_{P, \Omega^{*}}} u_{j, i}\right|_{\Omega^{*}}} \sum_{(j, i) \in I_{P, \Omega^{*}}} 2^{2 j}\right\| u_{j, i} \|_{L_{2}\left(\Omega^{*}\right)}^{2} \asymp \sum_{j=1}^{J} a_{j}^{2} 2^{-j(d-2)},
$$




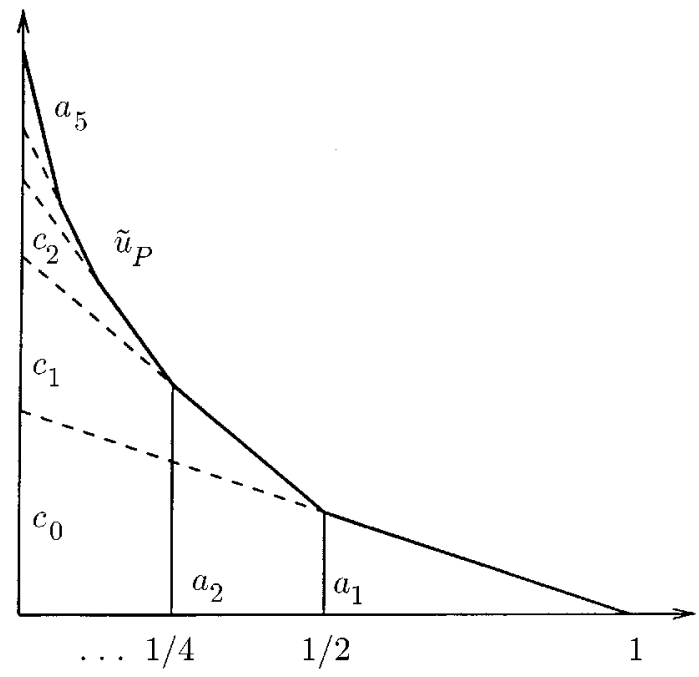

a)

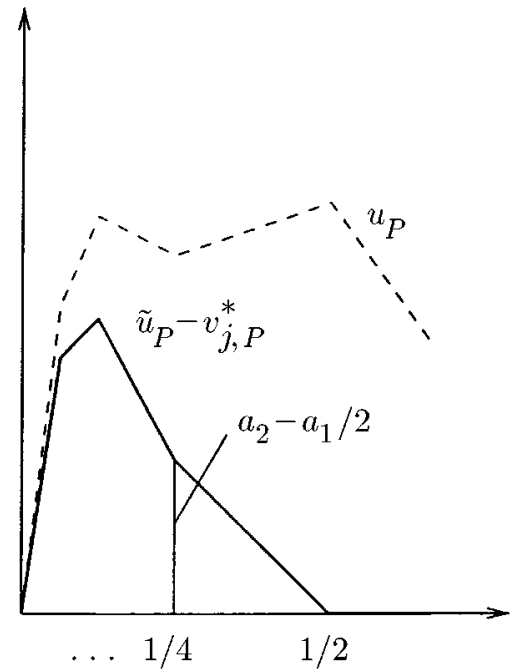

b)

FigURE 4. Illustration for a) $\tilde{u}_{P}$, and b) $u_{P}$ and $u_{P}-v_{j, P}^{*}$.

where

$$
a_{j}=\sum_{l=0}^{j-1}\left(1-2^{l-j}\right) c_{l}, \quad j=1, \ldots, J .
$$

Proof. Without loss of generality, let $\Omega^{*}$ be the simplex with vertices $P=(0,0, \ldots, 0), P_{1}=(1,0, \ldots, 0), P_{2}=(1,1,0, \ldots, 0), \ldots, P_{d}=(1,1, \ldots, 1)$.

Obviously, $u_{P}\left(x_{1}, x_{2}, \ldots, x_{d}\right)=\tilde{u}_{P}\left(x_{1}\right)$, where $\tilde{u}_{P}$ is a univariate linear spline function with respect to the partition $0<2^{-J}<2^{-J+1}<\ldots<2^{-1}<1$ determined by the values

$$
\tilde{u}_{P}(0)=a_{J+1}=\sum_{l=0}^{J} c_{l}, \tilde{u}_{P}\left(2^{-j}\right)=a_{j}, j=1, \ldots, J, \tilde{u}_{P}(1)=a_{0}=0 .
$$

Figure 4 a) illustrates the notation for $J=4$. Since $\left.u_{P}\right|_{\Omega^{*}}$ is constant along hyperplanes $x_{1}=$ const and vanishes at the $(d-1)$-dimensional face $x_{1}=1$, we have

$$
\left\|u_{P}\right\|_{1, \Omega^{*}}^{2} \asymp\left|u_{P}\right|_{1, \Omega^{*}}^{2} \asymp \sum_{j=0}^{J}\left(a_{j+1}-a_{j}\right)^{2} 2^{-j(d-2)} .
$$

Now, denote

$$
\begin{gathered}
b_{j}=a_{j+1}-a_{j}=2^{-1} c_{j}+2^{-2} c_{j-1}+\ldots+2^{-j-1} c_{0}, \quad j=0, \ldots, J-1, \\
b_{J}=2^{-1}\left(a_{J+1}-a_{J}\right)=2^{-1} c_{J}+2^{-2} c_{J-1}+\ldots+2^{-J-1} c_{0}, \quad b_{-1}=0,
\end{gathered}
$$

and observe that $2 b_{j}-b_{j-1}=c_{j}, j=0, \ldots, J$. These formulae give

$$
\sum_{j=0}^{J} 2^{-j(d-2)} c_{j}^{2} \asymp \sum_{j=0}^{J} 2^{-j(d-2)} b_{j}^{2}
$$


for all $d \geq 1$, which leads to (19).

To establish (20), we first remark that

$$
\|u\|\left\|_{P, \Omega^{*}}^{2} \asymp\right\| u \|_{L_{2}\left(\Omega^{*}\right)}^{2}+\sum_{j=1}^{J-1} 2^{2 j} E_{j}^{*}(u)_{L_{2}\left(\Omega^{*}\right)}^{2} \quad \forall u \in V_{J}^{*},
$$

where

$$
E_{j}^{*}(u)_{L_{2}\left(\Omega^{*}\right)}=\inf _{v_{j}^{*} \in V_{j}^{*}}\left\|u-v_{j}^{*}\right\|_{L_{2}\left(\Omega^{*}\right)}
$$

are the best approximations of $u$ in the $L_{2}\left(\Omega^{*}\right)$ norm with respect to $V_{j}^{*}$. The proof of (21) is the same as for the corresponding norm equivalence of Lemma 1 ; cf. [7, Theorem 6] and the remarks after Lemma 1.

We use (21) for $u=u_{P} \in V_{J}^{*}$ and the fact that $a_{J+1}=u_{P}(P)=0$. Let $v_{j, P}^{*} \in V_{j}^{*}$ denote the unique function which coincides with $u_{P}$ for $x_{1} \geq 2^{-j}$. We have the following trivial estimates:

$$
\left\|u_{P}\right\|_{L_{2}\left(\Omega^{*}\right)}^{2} \asymp \sum_{l=1}^{J} 2^{-d l} a_{l}^{2}
$$

and

$$
\begin{aligned}
E_{j}^{*}\left(u_{P}\right)_{L_{2}\left(\Omega^{*}\right)}^{2} & \leq\left\|u_{P}-v_{j, P}^{*}\right\|_{L_{2}\left(\Omega^{*}\right)}^{2}=\left\|u_{P}-v_{j, P}^{*}\right\|_{L_{2}\left(\Omega_{j}^{*}\right)}^{2} \\
& \leq 2\left(\left\|u_{P}\right\|_{L_{2}\left(\Omega_{j}^{*}\right)}^{2}+\left\|v_{j, P}^{*}\right\|_{L_{2}\left(\Omega_{j}^{*}\right)}^{2}\right) \leq C \sum_{l=j}^{J} 2^{-d l} a_{l}^{2},
\end{aligned}
$$

where $\Omega_{j}^{*}=\left\{x \in \Omega^{*}: x_{1} \leq 2^{-j}\right\}$. This leads to the upper estimate

$$
\left\|u_{P}\right\|_{P, \Omega^{*}}^{2} \leq C \sum_{j=0}^{J-1} 2^{2 j} \sum_{l=j}^{J} 2^{-d l} a_{l}^{2} \leq C \sum_{l=1}^{J} 2^{-d l} a_{l}^{2} \sum_{j=1}^{l} 2^{2 j} \leq C \sum_{j=1}^{J} 2^{-(d-2) j} a_{j}^{2} .
$$

On the other hand, we have

$$
E_{j}^{*}\left(u_{P}\right)_{L_{2}\left(\Omega^{*}\right)}^{2}=E_{j}^{*}\left(u_{P}-v_{j, P}^{*}\right)_{L_{2}\left(\Omega^{*}\right)}^{2} \geq c 2^{-d j}\left(a_{j+1}-a_{j} / 2\right)^{2}, \quad j=1, \ldots, J-1,
$$

where we have used the fact that $u_{P}-v_{j, P}^{*}$ is constant along hyperplanes $x_{1}=$ const, is piecewise linear with respect to $x_{1}$, vanishes for $x_{1} \geq 2^{-j}$ and $x_{1}=0$, and takes the value $a_{j+1}-a_{j} / 2$ at $x_{1}=2^{-j-1}$. See Figure $4 \mathrm{~b}$ ) for an illustration in the case $j=1, J=4$. Thus,

$$
\left\|u_{P}\right\|_{P, \Omega^{*}}^{2} \geq c\left(\sum_{l=0}^{J} 2^{-d l} a_{l}^{2}+\sum_{j=1}^{J-1} 2^{-(d-2) j}\left(a_{j+1}-a_{j} / 2\right)^{2}\right) \geq c \sum_{j=1}^{J} 2^{-(d-2) j} b_{j}^{2},
$$

where this time $b_{1}=a_{1}$ and $b_{j}=a_{j}-a_{j-1} / 2, j=2, \ldots, J$. Since the matrix transforming the vector $\alpha$ with components $\alpha_{j}=2^{-j(d / 2-1)} a_{j}$ into the vector $\beta$ with components

$$
\beta_{j}=2^{-j(d / 2-1)} b_{j}= \begin{cases}\alpha_{j}, & j=1, \\ \alpha_{j}-2^{-d / 2} \alpha_{j-1}, & j=2, \ldots, J\end{cases}
$$


is strictly diagonally dominant, with the identity matrix as diagonal part, we get

$$
||\left|u_{P}\right| \|_{P, \Omega^{*}}^{2} \geq c \sum \beta_{j}^{2} \geq c \sum \alpha_{j}^{2} \geq c \sum_{j=1}^{J} 2^{-(d-2) j} a_{j}^{2},
$$

which together with the already established upper estimate gives (20).

Lemma 5 will be used to construct functions $u$ with large $\||u|\|_{\omega}^{2}$ compared to $a_{\omega}(u, u)$. Let us first consider $d=2$. According to Lemma 4 it follows that for a non-exceptional $\mathcal{T}_{0}$ at least one of the conditions $(\beta)$ or $(\gamma)$ is satisfied. If $(\gamma)$ holds then there are two triangles $\Delta=\Omega_{1}$ and $\Delta^{\prime}=\Omega_{2}$ from $\mathcal{T}_{0}$ with $\bar{\Omega}_{1} \cap \bar{\Omega}_{2}=\{P\}$, where $P$ is an interior vertex. Figure $3 \mathrm{c}$ ) shows such a situation.

We set $\omega_{1}=\omega_{2}=1$, and $\omega_{k}=\epsilon$ for $k \geq 3$, where $\epsilon$ is chosen sufficiently small to essentially eliminate the influence of all other triangles. Set

$$
\left.u\right|_{\bar{\Delta}^{\prime}}=0,\left.\quad u\right|_{\bar{\Delta}}=\left.\left(\sum_{j=1}^{[J / 2]} \phi_{j, P}-\sum_{j=[J / 2]+1}^{2[J / 2]} \phi_{j, P}\right)\right|_{\bar{\Delta}} ;
$$

outside $\bar{\Delta} \cup \bar{\Delta}^{\prime}$ any extension to a function in $V_{J}$ will do. By Lemma 5 , (19), for sufficiently small $\epsilon$ we have

$$
a_{\omega}(u, u) \asymp\|u\|_{1, \Delta}^{2} \asymp \sum_{j=1}^{J} 1=J .
$$

On the other hand, by the property (9) of the triple bar norm $\||\cdot|\|_{\omega}$ and the definition of $\||\cdot|\|_{P, \Omega^{*}}$ from (20), we have

$$
\||| u \mid\|_{\omega}^{2} \geq c \inf _{u=u_{0}+u_{P}+\tilde{u}}\left(a_{\omega}\left(u_{0}, u_{0}\right)+\left\|u_{P}\right\|_{1, \Omega}^{2}+\left\|\tilde { u } \left|\left\|_{P, \Delta}^{2}+|\|\tilde{u}\||_{P, \Delta^{\prime}}^{2}\right)\right.\right.\right.
$$

where $u_{0} \in V_{0}$,

$$
u_{P}=\sum_{j=1}^{J} x_{j} \phi_{j, P}, \quad \sum_{j=1}^{J} x_{j}=-u_{0}(P) \equiv x_{0},
$$

and

$$
\tilde{u}=\sum_{j=1}^{J} \sum_{i: \phi_{j, i}(P)=0} u_{j, i} .
$$

To see this inequality, collect the terms in (9) into groups corresponding to whether 1) $(j, i)$ is associated with a $\phi_{j, P}$ (the sum of these $u_{j, i}$ gives $\left.\left.u_{P}\right), 2\right)(j, i) \in I_{P, \Delta}$, 3) $(j, i) \in I_{P, \Delta^{\prime}}$, or 4$)(j, i)$ is one of the remaining indices. After this, apply (19) to the sum of terms in group 1) and neglect all terms from group 4). This gives the above estimate from below (the terms corresponding to groups 2) and 3) give exactly the triple bar norms for $\tilde{u}$ associated with $(P, \Delta)$ and $\left(P, \Delta^{\prime}\right)$, respectively).

The important observation is that $\tilde{u}=u-u_{P}-u_{0}$ when restricted to either $\Delta$ or $\Delta^{\prime}$ is essentially (up to a $V_{0}$-perturbation) of the form $\sum_{j=0}^{J} c_{j} \phi_{j, P}$ which allows us to apply (20). Let us consider $\Delta$ first. If $Q, R$ denote the other two vertices of 
$\Delta$, and if $u_{0}$ takes the values $x_{0}, y, z$ at $P, Q, R$, respectively, then

$$
\left.\tilde{u}\right|_{\Delta}=\underbrace{\sum_{j=1}^{[J / 2]}\left(1-x_{j}\right) \phi_{j, P}-\sum_{j=[J / 2]+1}^{2[J / 2]}\left(1+x_{j}\right) \phi_{j, P}-x_{0} \phi_{0, P}}_{\equiv u_{P, \Delta}}-y \phi_{0, Q}-z \phi_{0, R}
$$

By (21) we have

$$
\|\tilde{u} \mid\|_{P, \Delta}^{2} \asymp\|\tilde{u}\|_{L_{2}(\Delta)}^{2}+\sum_{j=1}^{J-1} 2^{2 j} E_{j}^{*}\left(u_{P, \Delta}\right)_{L_{2}(\Delta)}^{2} .
$$

Since $\tilde{u}$ coincides with the linear function $u_{0}$ on the set $\Delta \backslash \operatorname{supp} \phi_{1, P}$, we also have

$$
x_{0}^{2}+y^{2}+z^{2} \asymp\left\|u_{0}\right\|_{L_{2}(\Delta)}^{2} \leq C\|\tilde{u}\|_{L_{2}(\Delta)}^{2},
$$

and

$$
\left\|u_{P, \Delta}\right\|_{L_{2}(\Delta)}^{2} \leq 2\left(\|\tilde{u}\|_{L_{2}(\Delta)}^{2}+\left\|y \phi_{0, Q}+z \phi_{0, R}\right\|_{L_{2}(\Delta)}^{2}\right) \leq C\|\tilde{u}\|_{L_{2}(\Delta)}^{2} .
$$

Altogether, again using (21) this gives

$$
\left.|||\tilde{u}|\right|_{P, \Delta} ^{2} \asymp\left(x_{0}^{2}+y^{2}+z^{2}\right)+\||| u_{P, \Delta}||_{P, \Delta}^{2} .
$$

For $\Delta^{\prime}$, the same consideration leads to

$$
\left\|\tilde{u}\left|\left\|_{P, \Delta^{\prime}}^{2} \asymp\left(x_{0}^{2}+\bar{y}^{2}+\bar{z}^{2}\right)+\right\|\right|\right\| u_{P, \Delta^{\prime}} \|_{P, \Delta^{\prime}}^{2},
$$

where

$$
u_{P, \Delta^{\prime}}=-\sum_{j=0}^{J} x_{j} \phi_{j, P}
$$

After all, looking at the discrete representations (20) for $d=2$, one immediately obtains the following lower bound for any choice of $x_{j}$ satisfying $\sum_{j=0}^{J} x_{j}=0$ (and, therefore, for any choice of $u_{0}$ and $u_{P}$ ):

$$
\begin{aligned}
\|\tilde{u}\|\left\|_{P, \Delta}^{2}+\right\| \tilde{u}\|\|_{P, \Delta^{\prime}}^{2} \geq c \sum_{j=1}^{J}\left(\tilde{a}_{j, \Delta}^{2}+\tilde{a}_{j, \Delta^{\prime}}^{2}\right) \geq c \sum_{j=1}^{J}\left(\tilde{a}_{j, \Delta}-\tilde{a}_{j, \Delta^{\prime}}\right)^{2} \\
\geq c\left\|\left|\left\|\sum_{j=1}^{[J / 2]} \phi_{j, P}-\sum_{j=[J / 2]+1}^{2[J / 2]} \phi_{j, P}\right\|\right|_{P, \Delta}^{2}=c\right\|\|u\|_{P, \Delta}^{2} \geq c J^{3} .
\end{aligned}
$$

Here, $\tilde{a}_{j, \Delta}$ resp. $\tilde{a}_{j, \Delta^{\prime}}$ stand for the values $a_{j}$ corresponding to the restrictions of $\tilde{u}$ to $\Delta$ resp. to $\Delta^{\prime}$, and it is stressed that $\tilde{a}_{j, \Delta}-\tilde{a}_{j, \Delta^{\prime}}$ are exactly the $a_{j}$ corresponding to $\left.u\right|_{\Delta}$. The computation of the latter is simple, and gives the lower bound of order $J^{3}$. Note that a similar function $u_{P}^{*}=\sum_{j=1}^{J} \phi_{j, P}$ has been used in $[13,8]$ to prove the asymptotic exactness of the condition number estimates for the hierarchical basis method of Yserentant. Thus, after all, we have shown that for some function $u \in V_{J}$ and a specific set of weights

$$
\|\mid u\|_{\omega}^{2} \geq c J^{2} a_{\omega}(u, u) \quad \Longrightarrow \quad \lambda_{\min } \leq c J^{-2} .
$$

The same argumentation works for the case $(\beta)$; see Figure $3 \mathrm{~b}$ ). Let $\Delta=\Omega_{1}$ be the triangle with boundary vertex $P$ (and such that the edges of $\Delta$ emanating from $P$ are in the interior of $\Omega$ ). Then $\omega_{1}=1$ and $\omega_{k}=\epsilon, k \geq 2 ;\left.u\right|_{\bar{\Delta}}$ is the same as above and will be suitably extended to some $u \in V_{J}$. Once again, (22) holds for 
$\epsilon$ small enough, and since there are no $u_{P}$ and $u_{0}$ components associated with a boundary vertex (i.e., $x_{j}=0$ for all $j$ ), we have

$$
\||| u\|_{\omega}^{2} \geq c \mid\|u\|_{P, \Delta}^{2} \geq c J^{3}
$$

as before.

The case $d \geq 3$ is similar to the case $d=2$. However, if $\mathcal{T}_{0}$ is of point type, the counterexamples are much simpler (see also [11]). Indeed, if $\Omega_{1}$ is a tetrahedron with vertices $P, P_{1}, P_{2}, P_{3}$ and such that $\{P\} \subset \Gamma_{1} \subset\left\{P, P_{1}, P_{2}, P_{3}\right\}$, then we set

$$
\omega_{1}=1, \omega_{k}=\epsilon, k \geq 2,\left.\quad u\right|_{\Omega_{1}}=1-\phi_{J, P}-\phi_{J, P_{1}}-\phi_{J, P_{2}}-\phi_{J, P_{3}},
$$

plus suitable extension to a function $u \in V_{J}$ which is sufficient to show the result of Theorem 1. For $\epsilon$ sufficiently small, the function satisfies

$$
a_{\omega}(u, u) \asymp 2^{-J},
$$

which can be verified by direct calculation. On the other hand, as above we obtain

$$
\left\|| | u \left|\left\|_{\omega}^{2} \geq\left. c\left|\| \phi_{0, P}-\phi_{J, P}\right|\right|_{P, \Omega_{1}} ^{2} \geq c .\right.\right.\right.
$$

Again, (20) is the essential ingredient for carrying out the computations. This gives the necessary examples in Theorem 1 for $\mathcal{T}_{0}$ of point type:

$$
\|\mid u\|_{\omega}^{2} \geq c 2^{J} a_{\omega}(u, u) \quad \Longrightarrow \quad \lambda_{\min } \leq c 2^{-J} .
$$

It remains to consider $\mathcal{T}_{0}$ of edge type. According to Lemma $4(\delta),(\epsilon)$, the situation is very much the same as in the case $d=2$. For example, if $(\delta)$ is satisfied for $\Omega_{1}$ with boundary edge $e$, then the crucial counterexample is obtained as follows. Let $P$ be the midpoint of $e$ and define $\tilde{\phi}_{j, P}$ as the unique function in $V_{j}$ vanishing at nodal points outside $e$ and satisfying

$$
\left.\tilde{\phi}_{j, P}\right|_{e}=\left.\phi_{1, P}\right|_{e} .
$$

Now

$$
\omega_{1}=1, \omega_{k}=\epsilon, k \geq 2,\left.\quad u\right|_{\bar{\Omega}_{1}}=\sum_{j=1}^{[J / 2]} \tilde{\phi}_{j, P}-\sum_{j=[J / 2]+1}^{2[J / 2]} \tilde{\phi}_{j, P},
$$

etc. gives the counterexample. Roughly speaking, this is the same example as for $d=2$ in the case $(\beta)$ of Lemma 4 but "stretched" along $e$. The details are left to the reader.

The above examples are extremal in many other problems. For example, the examples show the sharpness of the logarithmic factor in the estimates obtained by Bramble and $\mathrm{Xu}[4$, Theorem 4.5] for $d=2$; see (16) for the formulation of their result in the notation of our paper. Alternatively, for $d=2$ (16) reads as follows:

$$
E_{j}(u)_{L_{2, \omega}(\Omega)}^{2} \equiv \inf _{v_{j} \in V_{j}}\left\|u-v_{j}\right\|_{L_{2, \omega}(\Omega)}^{2} \leq C 2^{-2 j}(J-j) a_{\omega}(u, u) \quad \forall u \in V_{J},
$$

where the constant $C$ is independent of $\omega, j=0,1, \ldots, J-1$, and $J$.

Theorem 4. Let $d=2,3$. Suppose that the initial partition $\mathcal{T}_{0}$ is not exceptional. Then the $L_{2, \omega}$ orthoprojections $Q_{j, \omega}$ cannot be uniformly bounded, with a constant independent of $\omega$ and $J$. More precisely, the robust, $\omega$-independent estimates (16) and (17) are asymptotically sharp with respect to $J$ for $d=2$ (estimate (16) or (25)) and $d=3$ (with $\mathcal{T}_{0}$ of point type (estimate (16)) or edge type (estimate (17)). 
We leave this as an exercise. For $d=3$ and partitions of point type, see [11]. To obtain the worst case estimate for $d=2$ (and $\mathcal{T}_{0}$ of edge type if $d=3$ ), use the same examples as above, and estimate the best approximations $E_{j}(u)_{L_{2, \omega}(\Omega)}$ from below (on $\Delta$ and $\Delta^{\prime}$, as shown in the proof of Lemma 5) for $j \asymp J / 2$. Note that the statement of Theorem 4 also shows the asymptotical sharpness of the logarithmic term in Theorem 4.7 of [4] if $\mathcal{T}_{0}$ is not exceptional.

We conclude with another inequality which is of interest in the theory of nonoverlapping domain decomposition algorithms for two-dimensional problems but also demonstrates the fact that certain non-integer Sobolev norms are not additive with respect to the domain of definition. Let $d=1$, and set $\Omega \equiv I=[-1,1], I_{1}=[0,1]$, $I_{2}=[-1,0]$. Let $\mathcal{T}_{j}$ be the uniform partition of $I$ with stepsize $2^{-k}$, and let $V_{j}(I)=S_{1}^{0}\left(\mathcal{T}_{j}\right)$ be the spaces of linear splines.

Theorem 5. We have the estimate

$$
\|u\|_{H^{1 / 2}(I)} \leq C J\left(\|u\|_{H^{1 / 2}\left(I_{1}\right)}+\|u\|_{H^{1 / 2}\left(I_{2}\right)}\right), \quad u \in V_{J}(I),
$$

where the factor $J$ cannot be improved asymptotically.

Proof. The upper estimate is well-known. We provide the counterexamples and sketch the proof of the lower bound. Let $\phi_{j, 0}$ denote the nodal basis functions in $V_{j}(I)$ associated with the origin $x=0$, and set

$$
u= \begin{cases}\sum_{j=1}^{[J / 2]} \phi_{j, 0}-\sum_{j=[J / 2]+1}^{2[J / 2]} \phi_{j, 0}, & x \in I_{1} \\ 0, & x \in I_{2}\end{cases}
$$

Obviously, $u \in V_{J}(I)$ with $u(0)=0,\|u\|_{H^{1 / 2}\left(I_{2}\right)}=0$, and

$$
\|u\|_{H^{1 / 2}\left(I_{1}\right)}^{2} \asymp \inf _{u_{j, I_{1}} \in V_{j}\left(I_{1}\right): u=\sum_{j=1}^{J} u_{j, I_{1}}} \sum_{j=0}^{J} 2^{j}\left\|u_{j, I_{1}}\right\|_{L_{2}\left(I_{1}\right)}^{2} \leq C J .
$$

The last estimation in (27) follows if one takes $u_{j, I_{1}}=\phi_{j, 0}$ for $1 \leq j \leq[J / 2]$, and $u_{j, I_{1}}=-\phi_{j, 0}$ for $[J / 2]<j \leq 2[J / 2]$. The norm equivalence is contained in $[7$, Theorem 15].

On the other hand, the norm equivalence in (27) applied to the interval $I$ implies the existence of a decomposition $u=\sum_{j=0}^{J} u_{j}, u_{j} \in V_{j}(I)$, such that

$$
\|u\|_{H^{1 / 2}(I)}^{2} \asymp \sum_{j=0}^{J} 2^{j}\left\|u_{j}\right\|_{L_{2}(I)}^{2} .
$$

Set $x_{j}=u_{j}(0), \tilde{u}_{0}=\sum_{j=0}^{J} x_{j} \phi_{j, 0}$, and introduce, in analogy with (20), the following triple bar norms:

$$
\|\|_{0, I_{k}}^{2} \equiv \inf _{u_{j, I_{k}} \in V_{j}\left(I_{k}\right): u_{j, I_{k}}(0)=0, u=\sum_{j=1}^{J} u_{j, I_{k}}} \sum_{j=0}^{J} 2^{j}\left\|u_{j, I_{k}}\right\|_{L_{2}\left(I_{k}\right)}^{2}, \quad k=1,2 .
$$

A moment's reflection shows that the inequality from below in (28) implies

$$
\|u\|_{H^{1 / 2}(I)}^{2} \geq c\left(\left.||\left|u-\tilde{u}_{0}\right|\right|_{0, I_{1}} ^{2}+\left|\left\|\tilde{u}_{0}|\||_{0, I_{2}}^{2}\right) \geq c\right|\|u \mid\|_{0, I_{1}}^{2}\right.
$$


by the triangle inequality (note that $\left.||\left|\tilde{u}_{0}\right|\right|_{0, I_{2}}=||\left|\tilde{u}_{0}\right| \|_{0, I_{1}}$ by symmetry). Now, the triple bar norm of functions of the form $\left.u\right|_{I_{1}}=\sum_{j=1}^{J} c_{j} \phi_{j, 0}\left(\sum_{j=1}^{J} c_{j}=0\right)$ can be expressed by a formula almost identical to (20) of Lemma 5 , with $(d-2)$ in the exponent replaced by $(d-1)$. This corresponds to the change of the factors $2^{2 j}$ in the decomposition norms for the $H^{1}$ case to the factors $2^{j}$ in the $H^{1 / 2}$ case. Since for our $u$ we get $a_{j} \asymp \min (j, J-j)$, this leads to a lower bound of

$$
\|u\|_{H^{1 / 2}(I)}^{2} \geq c \mid\|u\| \|_{0, I_{1}}^{2} \geq c J^{3}
$$

and proves the assertion.

The example also shows that extension by zero does not lead to a bounded extension operator for $H^{1 / 2}$ spaces. Indeed, the above function $u$ is zero on $[-1,0]$ and can be considered as the extension by zero of $\tilde{u}=\left.u\right|_{[0,1]}$ (since $\tilde{u}$ is in $V_{J}(0,1)$ and satisfies $\tilde{u}(0)=\tilde{u}(1)=0$, it also belongs to

$$
H^{1 / 2}(0,1) \cap H_{0}^{1}(0,1) \subset \tilde{H}^{1 / 2}(0,1)=\left\{f=\left.g\right|_{[0,1]}: g \in H^{1 / 2}(\mathbb{R}), \operatorname{supp} g \subset[0,1]\right\}
$$

which is another, smaller space of interest in applications to interface problems and boundary integral equations). Thus, the $H^{1 / 2}$ norm of this particular extension operator is $\geq c J$, even when restricted to $V_{J}(0,1) \cap H_{0}^{1}(0,1)$, which shows its unboundedness on all of $H^{1 / 2}(0,1)$ and $\tilde{H}^{1 / 2}(0,1)$.

\section{REFERENCES}

[1] J. H. Bramble, Multigrid methods, Pitman Research Notes in Mathematical Sciences 294, Longman Sci.\&Techn., Harlow, Essex, 1993. MR 95b:65002

[2] J. H. Bramble, J. E. Pasciak, J. Wang, J. Xu, Convergence estimates for product iterative methods with applications to domain decomposition, Math. Comp. 57 (1991), 1-22. MR 92d:65094

[3] J. H. Bramble, J. E. Pasciak, J. Xu, Parallel multilevel preconditioners, Math. Comp. 55 (1990) 1-22. MR 90k:65170

[4] J. H. Bramble, J. Xu, Some estimates for a weighted $L^{2}$ projection, Math. Comp. 56 (1991), 463-476. MR 91k:65140

[5] M. Dryja, Multilevel methods for elliptic problems with discontinuous coefficients in three dimensions, Proceedings Seventh International Conference on Domain Decomposition, Contemporary Mathematics, vol. 180 (D. E. Keyes, J. Xu, eds.), AMS, Providence, 1994, pp. 43-47. MR 95j:65137

[6] M. Dryja, M. V. Sarkis, O. B. Widlund, Multilevel Schwarz methods for elliptic problems with discontinuous coefficients in three dimensions, Numer. Math. 72 (1996), 313-348. MR 96h:65134

[7] P. Oswald, Multilevel finite element approximation : theory and application, Teubner Skripten zur Numerik, Teubner, Stuttgart, 1994. MR 95k:65110

[8] P. Oswald, On estimates for hierarchic basis representations of finite element functions, Forsch.-Ergebnisse FSU Jena, N/89/16, 1989.

[9] P. Oswald, Stable subspaces splittings for Sobolev spaces and their applications, Preprint Math/93/7, FSU Jena, September 1993.

[10] J. Wang, New convergence estimates for multilevel algorithms for finite-element approximations, J. Comput. Appl. Math. 50 (1994), 593-604. MR 95d:65105

[11] J. Xu, Counterexamples concerning a weighted $L_{2}$-projection into finite element spaces, Math. Comp. 57 (1991), 563-568. MR 92b:65090

[12] J. Xu, Iterative methods by space decomposition and subspace correction, SIAM Rev. 34 (1992), 581-613. MR 93k:65029

[13] H. Yserentant, On the multilevel splitting of finite element spaces, Numer. Math. 49 (1986), 379-412. MR 88d:65068 
[14] H. Yserentant, Old and new convergence proofs for multigrid methods, Acta Numerica 1993, Cambr. Univ. Press, Cambridge, 1993, pp. 285-326. MR 94i:65128

[15] X. Zhang, Multilevel additive Schwarz methods, Numer. Math. 63 (1992), 521-539. MR 93h:65047

Bell Laboratories, Lucent Technologies, 600 Mountain Ave., Rm. 2C-403, Murray HiLL, NJ 07974-0636

E-mail address: poswald@research.bell-labs.com 